\title{
Persistence and stability of the
} disease-free equilibrium in a stochastic epidemic model with imperfect vaccine

\section{Dianli Zhao* and Sanling Yuan*}

\section{"Correspondence:}

Tc_zhaodianli@139.com; math-ysling@163.com

College of Science, University of

Shanghai for Science and

Technology, Shanghai, 200093,

China

\begin{abstract}
This paper concerns the dynamics of a stochastic SIVR epidemic model with imperfect vaccine where, differently from the epidemic model with perfect vaccine, the vaccinated is perturbed by the noise. This difference is the main difficulty to be conquered to give the threshold $R_{0}^{S}$. Firstly, $R_{0}^{S}>1$ is proved to be sufficient for persistence in mean of the system. Then, three conditions for the disease to die out are given, which improve the previously known results on extinction of the disease. In case that the disease goes extinct, we show that the disease-free equilibrium is almost surely stable by using the nonnegative semimartingale convergence theorem.
\end{abstract}

Keywords: stochastic epidemic model; imperfect vaccine; threshold value; stability

\section{Introduction}

Up to now, there are a lot of literatures on studying the disease showing that the deterministic and stochastic differential equations are often effective tools in describing the spread of a disease in real world; for more information, we refer to [1-5] and the references therein. The related known results also show us that finding the threshold value for the stochastic epidemic systems is extremely difficult and exciting; see, for example, [6-10].

Recently, inspired by works [8, 9], Tornatore et al. [10] formulated and studied a stochastic SIVR epidemic model by assuming that the average number of contacts per infective per unit time $\beta$ is perturbed by environmental noise with $\beta \rightarrow \beta+\sigma \dot{B}(t)$, where $B(t)$ is a standard Brownian motion on complete probability space $\left(\Omega, \mathcal{F},\left(\mathcal{F}_{t}\right)_{t \geq 0}, P\right)$ with intensity $\sigma^{2}>0$. This stochastic SIVR model reads as follows:

$$
\left\{\begin{array}{l}
d S=[\mu-(\mu+\phi) S-\beta S I] d t-\sigma S I d B(t), \\
d I=[\beta(S+\eta V) I-(\mu+\gamma) I] d t+\sigma(S+\eta V) I d B(t), \\
d V=(\phi S-\eta \beta V I-\mu V) d t-\sigma \eta V I d B(t), \\
d R=(\gamma I-\mu R) d t,
\end{array}\right.
$$

where the population is divided into four classes: susceptible, infective, vaccinated, and removed, denoted by $S, I, V$, and $R$, respectively; $\mu$ means the natural death rate of $S$, $I, V$, and $R$ compartments; the birth occurs in the system with the same rate of death;

(c) 2016 Zhao and Yuan. This article is distributed under the terms of the Creative Commons Attribution 4.0 International License (http://creativecommons.org/licenses/by/4.0/), which permits unrestricted use, distribution, and reproduction in any medium, provided you give appropriate credit to the original author(s) and the source, provide a link to the Creative Commons license, and indicate if changes were made. 
and $\gamma$ shows the rate of the infective going back in the susceptible class. It is assumed that, in the unit time, a fraction $\phi$ of the susceptible class is vaccinated. Considering that the vaccination may reduce, but not completely eliminate, susceptibility to infection, a factor $\eta(0 \leq \eta \leq 1)$ is introduced, where $\eta=0$ means that the vaccine is perfectly effective, and $\eta=1$ means that the vaccine has no effect. Throughout the paper, we assume that all parameter values are nonnegative and $\mu>0$. In [10], the authors have shown that the model permits solutions that are almost surely positive, and a theorem on exponential stability in mean square is proved under the condition

$$
\frac{\beta}{\mu+\gamma}+\frac{(1+\eta) \sigma^{2}}{2} \leq \frac{1}{1+\eta}
$$

Subsequently to the work of [3], Witbooi et al. [11] verified that the disease-free equilibrium point is almost surely exponentially stable if $\frac{\beta}{\mu+\gamma}<1$, which improves condition (2). However, for model (1), there are two basic and interesting questions yet to be answered:

Question A Under what conditions, will the disease be persistent?

Question B Does there exist a threshold under suitable condition, which is above one or below one, completely determining the persistence and extinction of the disease?

Considering these factors, in this paper, we focus our attention on model (1) to (I): obtaining explicit conditions that can ensure the disease to be persistent; (II): further developing the conditions for the infectious class to be extinct. Then, by comparison we give a threshold whose value below one or above one can determine the extinction and persistence of the epidemic under a mild extra condition. Finally, the obtained results are extended to the model studied by Tornatore et al. [12].

Comparing with models studied in $[6,7]$, the main difference is that the vaccination number considered in this paper is perturbed by the Brownian noise. This difference is the main difficulty to be conquered in establishing the threshold for model (1), which, to the best of our knowledge, has never been discussed in the previously known literature.

Remark 1 Tornatore et al. [3] have proved that system (1) has a unique positive solution $(S(t), I(t), V(t), R(t))$ on $t \geq 0$, with probability 1 , for any initial value $(S(0), I(0), V(0)$, $R(0)) \in R_{+}^{4}$.

From Remark 1 it is clear that a positive invariant set of system (1) can be given by

$$
\Gamma=\{(S, I, V, R): S>0, I>0, V>0, R>0, S+I+V+R \leq 1 \text { a.s. }\} .
$$

Without loss of generality, we set $S+I+V+R=1$. In the sequel, we denote $\langle x(t)\rangle=$ $\frac{1}{t} \int_{0}^{t} x(s) d s$. Define the important parameter

$$
R_{0}^{S}=\frac{1}{\mu+\gamma}\left[\beta \frac{\mu+\eta \phi}{\mu+\phi}-\frac{\sigma^{2}}{2}\left(\frac{\mu+\eta \phi}{\mu+\phi}\right)^{2}\right]
$$

The rest of the paper is organized as follows. In Section 2, we prove that the disease will persist if $R_{0}^{S}>1$. For $R_{0}^{S}$ below 1, in Section 3, we show that the disease goes extinct ex- 
ponentially when the noise is small. Then, by using the nonnegative semimartingale convergence theorem, we prove the stability of the disease-free equilibrium. In Section 4, the obtained results are extended to study the threshold value of a stochastic SIVS epidemic model. Finally, numerical simulations are introduced to support the obtained results.

\section{Persistence in mean}

Theorem 2.1 Let $(S(t), I(t), V(t), R(t))$ be a solution of system (1) with initial value $(S(0), I(0), V(0), R(0)) \in \Gamma$. If $R_{0}^{S}>1$, then the disease will be persistent in mean, that is,

$$
\liminf _{t \rightarrow \infty}\{I(t)\rangle \geq \frac{\mu+\gamma}{D}\left(R_{0}^{S}-1\right)>0 \quad \text { a.s. }
$$

where $D=\frac{\sigma^{2}}{2}\left(\frac{\phi(1-\eta) \eta(\mu+\gamma)}{\mu^{2}}+\frac{\sigma^{2}}{\mu}+\frac{\beta \phi(1-\eta)^{2}}{\mu(\mu+\phi)}+\beta \frac{\phi^{2}\left(1-\eta^{2}\right)+2 \mu \phi(1-\eta)}{\mu(\mu+\phi)}\right)+\beta\left(\eta+\frac{\eta \gamma}{\mu}+\frac{(1-\eta) \beta}{\mu+\phi}\right)$. Moreover,

$$
\begin{aligned}
& \liminf _{t \rightarrow \infty} \frac{1}{t} \int_{0}^{t} S(s) d s \geq \frac{\mu}{\mu+\gamma+\beta} \quad \text { a.s., } \\
& \liminf _{t \rightarrow \infty} \frac{1}{t} \int_{0}^{t} V(s) d s \geq \frac{\phi \mu}{(\mu+\eta \beta)(\mu+\gamma+\beta)} \quad \text { a.s., }
\end{aligned}
$$

and

$$
\liminf _{t \rightarrow \infty} \frac{1}{t} \int_{0}^{t} R(s) d s \geq \frac{\gamma(\mu+\gamma)}{D \mu}\left(R_{0}^{S}-1\right) \text { a.s. }
$$

To prove Theorem 2.1, we firstly prepare some lemmas.

Lemma 2.2 (See, e.g., [13], Lemma 2.1) Let $M(t), t \geq 0$, be a continuous local martingale with $M(0)=0$. Let $\theta>1$, and let $v_{k}$ and $\gamma_{k}$ be two sequences of positive numbers with $v_{k} \rightarrow \infty$. Then, for almost all $\omega \in \Omega$, there exists a random integer $k_{0}=k_{0}(\omega)$ such that, for all $k \geq k_{0}$,

$$
M(t) \leq \frac{1}{2} \gamma_{k}\langle M, M\rangle(t)+\frac{\theta}{\gamma_{k}} \ln k, \quad 0 \leq t \leq v_{k},
$$

where $\langle M, M\rangle(t)$ is the quadratic variation of $M(t)$.

Lemma 2.3 Let $g(t)$ be a continuous bounded function on $[0, \infty)$. Then

$$
\limsup _{t \rightarrow \infty} \frac{1}{\sqrt{t} \ln t}\left|\int_{0}^{t} g(s) d B(s)\right| \leq 1 \quad \text { a.s. }
$$

and, for any constant $\xi>0$,

$$
\limsup _{t \rightarrow \infty} \frac{1}{\sqrt{t} \ln t}\left|\int_{0}^{t} g(s) e^{-\xi(t-s)} d B(s)\right| \leq 1 \quad \text { a.s. }
$$

Proof By choosing $\gamma_{k}=\frac{1}{\sqrt{k}}, v_{k}=k$, and $\theta=1$ in Lemma 2.2, for sufficiently large $k$ and $k-1<t \leq k$, we have

$$
\int_{0}^{t} g(s) d B(s) \leq \frac{1}{2 \sqrt{k}} \int_{0}^{t} g^{2}(s) d s+\sqrt{k} \ln k \leq \frac{t}{2 \sqrt{k}} \sup _{s \geq 0}\left\{g^{2}(s)\right\}+\sqrt{k} \ln k \quad \text { a.s. }
$$


By applying Lemma 2.2 to $-\int_{0}^{t} g(s) d B(s)$ we have

$$
-\int_{0}^{t} g(s) d B(s) \leq \frac{t}{2 \sqrt{k}} \sup _{s \geq 0}\left\{g^{2}(s)\right\}+\sqrt{k} \ln k \quad \text { a.s. }
$$

Then, it follows that

$$
\left|\int_{0}^{t} g(s) d B(s)\right| \leq \frac{t}{2 \sqrt{k}} \sup _{s \geq 0}\left\{g^{2}(s)\right\}+\sqrt{k} \ln k \quad \text { a.s. }
$$

Noting that $k-1<t \leq k$, this yields that

$$
\sup _{k-1<t \leq k}\left\{\left|\int_{0}^{t} g(s) d B(s)\right|\right\} \leq \frac{\sqrt{t}}{2} \sup _{s \geq 0}\left\{g^{2}(s)\right\}+\sqrt{t+1} \ln (t+1) \quad \text { a.s. }
$$

This leads to

$$
\limsup _{t \rightarrow \infty} \frac{\left|\int_{0}^{t} g(s) d B(s)\right|}{\sqrt{t} \ln t} \leq \limsup _{t \rightarrow \infty} \frac{\frac{\sqrt{t}}{2} \sup _{s \geq 0}\left\{g^{2}(s)\right\}+\sqrt{t+1} \ln (t+1)}{\sqrt{t} \ln t}=1 \text { a.s. }
$$

To prove (5), we choose $\gamma_{k}=\frac{e^{-\xi(k-1)}}{\sqrt{k}}, v_{k}=k$, and $\theta=1$ in Lemma 2.2. Then, for $k-1<$ $t \leq k$,

$$
\begin{aligned}
& \left|\int_{0}^{t} g(s) e^{-\xi(t-s)} d B(s)\right| \\
& \quad=e^{-\xi t}\left|\int_{0}^{t} g(s) e^{\xi s} d B(s)\right| \leq e^{-\xi t}\left(\frac{1}{2} \frac{e^{-\xi(k-1)}}{\sqrt{k}} \int_{0}^{t} g^{2}(s) e^{2 \xi s} d s+e^{\xi(k-1)} \sqrt{k} \ln k\right) \\
& \quad \leq e^{-\xi t}\left(\frac{e^{-\xi(k-1)} e^{2 \xi t}}{4 \xi \sqrt{k}} \sup _{s \geq 0}\left\{g^{2}(s)\right\}+e^{\xi(k-1)} \sqrt{k} \ln k\right) \\
& \quad \leq \frac{e^{\xi}}{4 \xi \sqrt{t}} \sup _{s \geq 0}\left\{g^{2}(s)\right\}+\sqrt{t+1} \ln (t+1) \quad \text { a.s. }
\end{aligned}
$$

It follows that

$$
\limsup _{t \rightarrow \infty} \frac{\left|\int_{0}^{t} g(s) e^{-\xi(t-s)} d B(s)\right|}{\sqrt{t} \ln t} \leq \limsup _{t \rightarrow \infty} \frac{\frac{e^{\xi}}{4 \xi \sqrt{t}} \sup _{s \geq 0}\left\{g^{2}(s)\right\}+\sqrt{t+1} \ln (t+1)}{\sqrt{t} \ln t}=1 \quad \text { a.s. }
$$

The proof is complete.

Lemma 2.4 Let $(S(t), I(t), V(t), R(t))$ be a solution of system $(1)$ with initial value $(S(0), I(0)$, $V(0), R(0)) \in \Gamma$. Then

$$
S(t)+\eta V(t)=\frac{\mu+\eta \phi}{\mu+\phi}-H(t, I(t))+\varphi(t)-(1-\eta) \sigma \int_{0}^{t} S(s) I(s) e^{-(\mu+\phi)(t-s)} d B(s),
$$

where

$$
H(t, I(t))=\left(\eta I+\eta \gamma \int_{0}^{t} I(s) e^{-\mu(t-s)} d s+(1-\eta) \beta \int_{0}^{t} S(s) I(s) e^{-(\mu+\phi)(t-s)} d s\right)
$$


and

$$
\varphi(t)=-(1-\eta)\left(\frac{\mu}{\mu+\phi}-S(0)\right) e^{-(\mu+\phi) t} .
$$

Proof From (1) we get

$$
R(t)=R(0) e^{-\mu t}+\gamma \int_{0}^{t} I(s) e^{-\mu(t-s)} d s
$$

and

$$
\begin{aligned}
S(t)= & \frac{\mu}{\mu+\phi}-\left(\frac{\mu}{\mu+\phi}-S(0)\right) e^{-(\mu+\phi) t}-\beta \int_{0}^{t} S(s) I(s) e^{-(\mu+\phi)(t-s)} d s \\
& -\sigma \int_{0}^{t} S(s) I(s) e^{-(\mu+\phi)(t-s)} d B(s) .
\end{aligned}
$$

Then we can compute that

$$
\begin{aligned}
S(t)+ & \eta V(t) \\
= & \eta(S(t)+V(t))+(1-\eta) S(t) \\
= & \eta\left(1-[S(0)+I(0)+V(0)+R(0)-1] e^{-\mu t}-I(t)-R(0) e^{-\mu t}-\gamma \int_{0}^{t} I(s) e^{-\mu(t-s)} d s\right) \\
& +(1-\eta)\left[\frac{\mu}{\mu+\phi}-\left(\frac{\mu}{\mu+\phi}-S(0)\right) e^{-(\mu+\phi) t}\right] \\
& -(1-\eta)\left[\beta \int_{0}^{t} S(s) I(s) e^{-(\mu+\phi)(t-s)} d s+\sigma \int_{0}^{t} S(s) I(s) e^{-(\mu+\phi)(t-s)} d B(s)\right] .
\end{aligned}
$$

The proof is complete.

Proof of Theorem 2.1 Applying Itô's formula to system (1) and then integrating lead to

$$
\frac{1}{t} \ln \frac{I(t)}{I(0)}=\beta\langle S(t)+\eta V(t)\rangle-(\mu+\gamma)-\frac{\sigma^{2}}{2}\left\langle(S(t)+\eta V(t))^{2}\right\rangle+\frac{\sigma M(t)}{t},
$$

where $M(t)=\int_{0}^{t}(S(s)+\eta V(s)) d B(s)$ is a martingale. By Lemma 2.4 we have

$$
\langle S(t)+\eta V(t)\rangle=\frac{\mu+\eta \phi}{\mu+\phi}-\langle H(t, I(t))\rangle-\langle\varphi(t)\rangle-(1-\eta) \sigma \frac{K_{1}(t)}{t},
$$

where

$$
\begin{aligned}
\langle H(t, I(t))\rangle & =\left\langle\eta I(t)+\eta \gamma \int_{0}^{t} I(s) e^{-\mu(t-s)} d s+(1-\eta) \beta \int_{0}^{t} S(s) I(s) e^{-(\mu+\phi)(t-s)} d s\right\rangle \\
& \leq\left(\eta+\frac{\eta \gamma}{\mu}+\frac{(1-\eta) \beta}{\mu+\phi}\right)\langle I(t)\rangle
\end{aligned}
$$

and

$$
K_{1}(t)=\int_{0}^{t} \int_{0}^{v} S(s) I(s) e^{-(\mu+\phi)(v-s)} d B(s) d v
$$


Next, we compute $\left\langle(S(t)+\eta V(t))^{2}\right\rangle$. From (1) we have

$$
\begin{aligned}
& d S=[\mu-(\mu+\phi) S-\beta S I] d t-\sigma S I d B(t), \\
& \begin{aligned}
d S^{2}=[2 \mu S- & \left.2(\mu+\phi) S^{2}-2 \beta S^{2} I+\sigma^{2} S^{2} I^{2}\right] d t-2 \sigma S^{2} I d B(t), \\
d(S+\eta V)=[ & \left.\mu-\mu(S+\eta V)-\phi(1-\eta) S-\beta S I-\eta^{2} \beta V I\right] d t-\sigma\left(S+\eta^{2} V\right) I d B(t), \\
d(S+\eta V)^{2}= & {\left[2 \mu(S+\eta V)-2 \mu(S+\eta V)^{2}-2 \phi(1-\eta)^{2} S^{2}-2 \phi(1-\eta) \eta S\right.} \\
& +2 \phi(1-\eta) \eta S(I+R)-2(S+\eta V)\left(\beta S+\eta^{2} \beta V\right) I \\
& \left.+\sigma^{2}\left(S+\eta^{2} V\right)^{2} I^{2}\right] d t-2 \sigma(S+\eta V)\left(S+\eta^{2} V\right) I d B(t) .
\end{aligned}
\end{aligned}
$$

The last equality is obtained by using $S(t)+I(t)+V(t)+R(t)=1$. Denoting

$$
\begin{aligned}
\Psi(t)= & \frac{(S(t)+\eta V(t))^{2}}{2 \mu}+\frac{S(t)+\eta V(t)}{\mu}-\frac{\phi(1-\eta)^{2}}{\mu} \frac{S^{2}(t)}{2(\mu+\phi)} \\
& -\left(\frac{\phi^{2}\left(1-\eta^{2}\right)+2 \mu \phi(1-\eta)}{\mu(\mu+\phi)}\right) \frac{S(t)}{\mu+\phi}
\end{aligned}
$$

it follows that

$$
\left\langle(S(t)+\eta V(t))^{2}\right\rangle=\left(\frac{\mu+\phi \eta}{\mu+\phi}\right)^{2}+\left\langle H_{1}(t, I(t))\right\rangle+\frac{M_{1}(t)}{t}-\frac{\Psi(t)-\Psi(0)}{t},
$$

where

$$
\begin{aligned}
M_{1}(t)= & \int_{0}^{t}\left[-\frac{\sigma}{\mu}(S(s)+\eta V(s))\left(S(s)+\eta^{2} V(s)\right)-\frac{\sigma}{\mu}\left(S(s)+\eta^{2} V(s)\right)+\frac{\phi(1-\eta)^{2} \sigma}{\mu(\mu+\phi)} S^{2}(s)\right. \\
& \left.+\left(\frac{\phi^{2}\left(1-\eta^{2}\right)+2 \mu \phi(1-\eta)}{\mu(\mu+\phi)}\right) \sigma S(s)\right] I(s) d B(s)
\end{aligned}
$$

and

$$
\begin{aligned}
H_{1}(t, I(t))= & \frac{\phi(1-\eta) \eta}{\mu} S(t)(I(t)+R(t))-\frac{1}{\mu}(S(t)+\eta V(t))\left(\beta S(t)+\eta^{2} \beta V(t)\right) I(t) \\
& +\frac{\sigma^{2}}{\mu}\left(S(t)+\eta^{2} V(t)\right)^{2} I^{2}(t)-\frac{\beta}{\mu} S(t) I(t)-\frac{\eta^{2} \beta}{\mu} V(t) I(t) \\
& +\frac{\beta \phi(1-\eta)^{2}}{\mu(\mu+\phi)} S^{2}(t) I(t)-\frac{\phi(1-\eta)^{2} \sigma^{2}}{2 \mu(\mu+\phi)} S^{2}(t) I^{2}(t) \\
& +\left(\frac{\phi^{2}\left(1-\eta^{2}\right)+2 \mu \phi(1-\eta)}{\mu(\mu+\phi)}\right) \beta S(t) I(t),
\end{aligned}
$$

so that

$$
\begin{aligned}
\left\langle H_{1}(t, I(t))\right\rangle \leq & {\left[\frac{\phi(1-\eta) \eta}{\mu}+\frac{\sigma^{2}}{\mu}+\frac{\beta \phi(1-\eta)^{2}}{\mu(\mu+\phi)}+\beta \frac{\phi^{2}\left(1-\eta^{2}\right)+2 \mu \phi(1-\eta)}{\mu(\mu+\phi)}\right]\langle I(t)\rangle } \\
& +\frac{\phi(1-\eta) \eta}{\mu}\langle R(t)\rangle
\end{aligned}
$$




$$
\begin{aligned}
\leq & {\left[\frac{\phi(1-\eta) \eta(\mu+\gamma)}{\mu^{2}}+\frac{\sigma^{2}}{\mu}+\frac{\beta \phi(1-\eta)^{2}}{\mu(\mu+\phi)}+\beta \frac{\phi^{2}\left(1-\eta^{2}\right)+2 \mu \phi(1-\eta)}{\mu(\mu+\phi)}\right] } \\
& \times\langle I(t)\rangle \\
& -\frac{\phi(1-\eta) \eta}{\mu} \frac{R(t)-R(0)}{t} .
\end{aligned}
$$

Combing (10) and (11)-(14), we obtain

$$
\begin{aligned}
\frac{1}{t} \ln \frac{I(t)}{I(0)}= & \beta \frac{\mu+\eta \phi}{\mu+\phi}-(\mu+\gamma)-\frac{\sigma^{2}}{2}\left(\frac{\mu+\phi \eta}{\mu+\phi}\right)^{2}-\beta\langle H(t, I(t))\rangle-\frac{\sigma^{2}}{2}\left\langle H_{1}(t, I(t))\right\rangle \\
& -\beta\langle\varphi(t)\rangle+\frac{\sigma^{2}}{2} \frac{\Psi(t)-\Psi(0)}{t}-(1-\eta) \sigma \beta \frac{K_{1}(t)}{t}-\frac{\sigma^{2}}{2} \frac{M_{1}(t)}{t}+\frac{\sigma M(t)}{t} \\
\geq & (\mu+\gamma)\left(R_{0}^{S}-1\right)-D\langle I(t)\rangle-\beta\langle\varphi(t)\rangle+\frac{\sigma^{2}}{2} \frac{\Psi(t)-\Psi(0)}{t} \\
& -\frac{\sigma^{2}}{2} \frac{\phi(1-\eta) \eta}{\mu} \frac{R(t)-R(0)}{t}-(1-\eta) \sigma \beta \frac{K_{1}(t)}{t} \\
& -\frac{\sigma^{2}}{2} \frac{M_{1}(t)}{t}+\frac{\sigma M(t)}{t} .
\end{aligned}
$$

Integration by parts yields

$$
K_{1}(t)=\frac{1}{\mu+\phi}\left(\int_{0}^{t} S(s) I(s) d B(s)-\int_{0}^{t} S(s) I(s) e^{-(\mu+\phi)(t-s)} d B(s)\right) .
$$

Noting that $(S(t), I(t), V(t), R(t)) \in \Gamma$ and using Lemma 2.3, we have that

$$
\begin{aligned}
& \lim _{t \rightarrow \infty}\langle\varphi(t)\rangle=0, \quad \lim _{t \rightarrow \infty} \frac{\Psi(t)-\Psi(0)}{t}=0, \quad \lim _{t \rightarrow \infty} \frac{R(t)-R(0)}{t}=0 ; \\
& \lim _{t \rightarrow \infty} \frac{K_{1}(t)}{t}=0, \quad \lim _{t \rightarrow \infty} \frac{M_{1}(t)}{t}=0, \quad \lim _{t \rightarrow \infty} \frac{M(t)}{t}=0 ; \quad \text { and } \\
& \limsup _{t \rightarrow \infty} \frac{1}{t} \ln \frac{I(t)}{I(0)} \leq \limsup _{t \rightarrow \infty} \frac{1}{t} \ln \frac{1}{I(0)} \leq 0 .
\end{aligned}
$$

These, together with (15), show us that

$$
\liminf _{t \rightarrow \infty}\langle I(t)\rangle \geq \frac{\mu+\gamma}{D}\left(R_{0}^{S}-1\right)>0 .
$$

Next, to prove that system (1) is persistent in mean, it remains to prove that $S, V$, and $R$ are persistent. From (1) we have

$$
\begin{aligned}
\frac{S(t)-S(0)}{t}+\frac{\sigma}{t} \int_{0}^{t} S(s) I(s) d B(s) & =\mu-(\mu+\phi)\langle S(t)\rangle-\beta\langle S(t) I(t)\rangle \\
\geq & \mu-(\mu+\phi+\beta)\langle S(t)\rangle \\
\frac{V(t)-V(0)}{t}+\frac{\sigma \eta}{t} \int_{0}^{t} V(s) I(s) d B(s) & =\phi\langle S(t)\rangle-\eta \beta V\langle V(t) I(t)\rangle-\mu\langle V(t)\rangle \\
& \geq \phi\langle S(t)\rangle-(\eta \beta+\mu)\langle V(t)\rangle,
\end{aligned}
$$


and

$$
\frac{R(t)-R(0)}{t}=\gamma\langle I(t)\rangle-\mu\langle R(t)\rangle
$$

By Lemma 2.3 these yield

$$
\begin{aligned}
& \liminf _{t \rightarrow \infty}\langle S(t)\rangle \geq \frac{\mu}{\mu+\phi+\beta} \quad \text { a.s., } \\
& \liminf _{t \rightarrow \infty}\langle V(t)\rangle \geq \frac{\phi \mu}{(\eta \beta+\mu)(\mu+\phi+\beta)} \quad \text { a.s., }
\end{aligned}
$$

and

$$
\liminf _{t \rightarrow \infty}\langle R(t)\rangle \geq \frac{\gamma}{\mu} \liminf _{t \rightarrow \infty}\langle I(t)\rangle \quad \text { a.s. }
$$

The proof is complete.

Remark 2 In Theorem 2.1, if $\phi>0$, then the population in any one of four states will be persistent, and system (1) is persistent in mean. If $\phi=0$, then the vaccination is perfectly effective, and the number of the vaccinated class tends to zero due to the effect of the environmental noise, and the obtained result is consistent with that in [6].

\section{Stability of the disease-free equilibrium}

Theorem 3.1 Let $(S(t), I(t), V(t), R(t))$ be a solution of system (1) with initial value $(S(0), I(0), V(0), R(0)) \in \Gamma$. Suppose that one of the following three assumptions holds:
(A) $\frac{\beta^{2}}{2 \sigma^{2}}<\mu+\gamma$
(B) $\beta \frac{\mu+\eta \phi}{\mu+\phi}-(\mu+\gamma)-\frac{\mu^{2} \sigma^{2}}{2(\mu+\phi+\beta)^{2}}<0$;
(C) $\sigma^{2} \frac{\mu+\eta \phi}{\mu+\phi} \leq \beta$ and $R_{0}^{S}<1$.

Then the disease almost surely exponentially dies out, that is,

$$
\begin{aligned}
& \limsup _{t \rightarrow \infty} \frac{1}{t} \ln \frac{I(t)}{I(0)} \leq \frac{\beta^{2}}{2 \sigma^{2}}-(\mu+\gamma)<0 \quad \text { a.s. if (A) holds; } \\
& \limsup _{t \rightarrow \infty} \frac{1}{t} \ln \frac{I(t)}{I(0)} \leq \beta \frac{\mu+\eta \phi}{\mu+\phi}-(\mu+\gamma)-\frac{\mu^{2} \sigma^{2}}{2(\mu+\phi+\beta)^{2}}<0 \quad \text { a.s. if (B) holds; } \\
& \limsup _{t \rightarrow \infty} \frac{1}{t} \ln \frac{I(t)}{I(0)} \leq(\mu+\gamma)\left(R_{0}^{S}-1\right)<0 \quad \text { a.s. if (C) holds. }
\end{aligned}
$$


Proof If (A) holds, then by (10) we have

$$
\begin{aligned}
\frac{1}{t} \ln \frac{I(t)}{I(0)} & \leq \beta\langle S(t)+\eta V(t)\rangle-(\mu+\gamma)-\frac{\sigma^{2}}{2}\langle(S(t)+\eta V(t))\rangle^{2}+\frac{\sigma M(t)}{t} \\
& \leq \frac{\beta^{2}}{2 \sigma^{2}}-(\mu+\gamma)-\frac{\sigma^{2}}{2}\left[\langle S(t)+\eta V(t)\rangle-\frac{\beta}{\sigma^{2}}\right]^{2}+\frac{\sigma M(t)}{t} \\
& \leq \frac{\beta^{2}}{2 \sigma^{2}}-(\mu+\gamma)+\frac{\sigma M(t)}{t}
\end{aligned}
$$

The desired result can be derived by using (17).

If (B) holds, then by (10)

$$
\begin{aligned}
\frac{1}{t} \ln \frac{I(t)}{I(0)} & =\beta\langle S(t)+\eta V(t)\rangle-(\mu+\gamma)-\frac{\sigma^{2}}{2}\left\langle(S(t)+\eta V(t))^{2}\right\rangle+\frac{\sigma M(t)}{t} \\
& \leq \beta \frac{\mu+\eta \phi}{\mu+\phi}-(\mu+\gamma)-\frac{\sigma^{2}}{2}\langle S(t)\rangle^{2}+\frac{\sigma M(t)}{t}-\beta\langle\varphi(t)\rangle-(1-\eta) \sigma \beta \frac{K_{1}(t)}{t} .
\end{aligned}
$$

By applying (16), (17), and (18) we get

$$
\limsup _{t \rightarrow \infty} \frac{1}{t} \ln \frac{I(t)}{I(0)} \leq \beta \frac{\mu+\eta \phi}{\mu+\phi}-(\mu+\gamma)-\frac{\mu^{2} \sigma^{2}}{2(\mu+\phi+\beta)^{2}}<0 .
$$

If (C) holds, then by (10)

$$
\begin{aligned}
\frac{1}{t} \ln \frac{I(t)}{I(0)} \leq & \beta\langle S(t)+\eta V(t)\rangle-(\mu+\gamma)-\frac{\sigma^{2}}{2}\langle S(t)+\eta V(t)\rangle^{2}+\frac{\sigma M(t)}{t} \\
\leq & \beta \frac{\mu+\eta \phi}{\mu+\phi}-(\mu+\gamma)-\frac{\sigma^{2}}{2}\left(\frac{\mu+\eta \phi}{\mu+\phi}\right)^{2}+\left(\sigma^{2} \frac{\mu+\eta \phi}{\mu+\phi}-\beta\right)\langle H(t, I(t))\rangle \\
& +\Phi(t),
\end{aligned}
$$

where

$$
\Phi(t)=2\left(\frac{\mu+\eta \phi}{\mu+\phi}-\langle H(t, I(t))\rangle\right)\left[\langle\varphi(t)\rangle-(1-\eta) \sigma \frac{K_{1}(t)}{t}\right]+\left[\langle\varphi(t)\rangle-(1-\eta) \sigma \frac{K_{1}(t)}{t}\right]^{2} .
$$

Noting that (16) and (17) imply $\lim _{t \rightarrow \infty} \Phi(t)=0$, we have

$$
\limsup _{t \rightarrow \infty} \frac{1}{t} \ln \frac{I(t)}{I(0)} \leq(\mu+\gamma)\left(R_{0}^{S}-1\right)<0 \quad \text { a.s. }
$$

In summary, if one of the three assumptions holds, then the disease will die out exponentially. The proof is complete.

Theorem 3.2 Suppose that the conditions in Theorem 3.1 hold. Then the disease-free equilibrium point $\left(S_{0}, I_{0}, V_{0}, R_{0}\right)=\left(\frac{\mu}{\mu+\phi}, 0, \frac{\phi}{\mu+\phi}, 0\right)$ is almost surely stable, namely,

$$
(S(t), I(t), V(t), R(t)) \rightarrow\left(S_{0}, I_{0}, V_{0}, R_{0}\right) \quad \text { a.s. as } t \rightarrow \infty \text {. }
$$

The following results are needed for the proof of Theorem 3.2. 
Lemma 3.3 (See, e.g., [14], Thm. 3.9) Let $A(t)$ and $U(t)$ be two continuous adapted increasing process on $t \geq 0$ with $A(0)=U(0)=0$ a.s. Let $M(t)$ be a real-valued continuous local martingale with $M(0)=0$ a.s. Let $X_{0}$ be a nonnegative $\mathcal{F}_{0}$-measurable random variable such that $E X_{0}<\infty$. Define $X(t)=X_{0}+A(t)-U(t)+M(t)$ for all $t \geq 0$. If $X(t)$ is nonnegative, then $\lim _{t \rightarrow \infty} A(t)<\infty$ implies that $\lim _{t \rightarrow \infty} U(t)<\infty, \lim _{t \rightarrow \infty} X(t)<\infty$, and $-\infty<\lim _{t \rightarrow \infty} M(t)<\infty$ a.s.

Consider the equation

$$
x(t)=x\left(t_{0}\right)+\int_{t_{0}}^{t} f(s, x(s)) d s+\int_{t_{0}}^{t} g(s, x(s)) d \widetilde{B}(s), \quad x \in R^{n}, t_{0} \in R .
$$

Here, $\widetilde{B}(s)$ is an $m$-dimensional Brownian motion.

Lemma 3.4 (See, e.g., [15], Lemma 2.4) Suppose that:

(C1) The functions $f$ and $g$ satisfy the local Lipschitz and linear growth conditions;

(C2) $\sup _{t \geq 0}\left\{E|x(t)|^{p}\right\}<\infty$, where $|\cdot|$ is the Euclidean norm in $R^{n}$.

Then almost every sample path of $\int_{0}^{t} g(s, x(s)) d \tilde{B}(s)$ is uniformly continuous on $t \geq 0$.

Lemma 3.5 (See, e.g., [16], Lemma 2.1) Let h be a nonnegative function defined on $[0, \infty)$ that is integrable on $[0, \infty)$ and uniformly continuous on $[0, \infty)$. Then $\lim _{t \rightarrow \infty} h(t)=0$.

Proof By applying Itô's formula to the first equation in (1),

$$
\begin{aligned}
d\left(\frac{\mu}{\mu+\phi}-S\right)^{2}= & {\left[-2(\mu+\phi)\left(\frac{\mu}{\mu+\phi}-S\right)^{2}+2 \beta S I\left(\frac{\mu}{\mu+\phi}-S\right)+\sigma^{2} S^{2} I^{2}\right] d t } \\
& +2 \sigma S I\left(\frac{\mu}{\mu+\phi}-S\right) d B(t) .
\end{aligned}
$$

Then integrating this formula and rearranging the expression, we have

$$
\begin{aligned}
\int_{0}^{t} & \left(\frac{\mu}{\mu+\phi}-S(s)\right)^{2} d s \\
= & \frac{1}{2(\mu+\phi)}\left[S(t)-S(0)+2 \beta \int_{0}^{t} S(s) I(s)\left(\frac{\mu}{\mu+\phi}-S(s)\right) d s\right] \\
& +\frac{1}{2(\mu+\phi)} \int_{0}^{t} \sigma^{2} S^{2}(s) I^{2}(s) d s+\frac{\sigma}{\mu+\phi} \int_{0}^{t} \beta S(s) I(s)\left(\frac{\mu}{\mu+\phi}-S(s)\right) d B(s) \\
\leq & \frac{1}{2(\mu+\phi)}\left[1+2 \beta \frac{\mu}{\mu+\phi} \int_{0}^{t} I(s) d s+\sigma^{2} \int_{0}^{t} I^{2}(s) d s\right]+\tilde{M}(t)
\end{aligned}
$$

where $\tilde{M}(t)=\frac{\beta \sigma}{\mu+\phi} \int_{0}^{t} S(s) I(s)\left(\frac{\mu}{\mu+\phi}-S(s)\right) d B(s)$ is a continuous local martingale. Let

$$
X(t)=\frac{1}{2(\mu+\phi)}\left[1+2 \beta \frac{\mu}{\mu+\phi} \int_{0}^{t} I(s) d s+\sigma^{2} \int_{0}^{t} I^{2}(s) d s\right]+\tilde{M}(t)
$$


with $X(0)=\frac{1}{2(\mu+\phi)}$. Clearly, $X(t) \geq \int_{0}^{t}\left(\frac{\mu}{\mu+\phi}-S(s)\right)^{2} d s \geq 0$. By Theorem 3.1, $I(t)$ exponentially tends to 0 , which implies that

$$
\frac{1}{2(\mu+\phi)}\left[2 \beta \frac{\mu}{\mu+\phi} \int_{0}^{t} I(s) d s+\sigma^{2} \int_{0}^{t} I^{2}(s) d s\right]<\infty \quad \text { a.s. }
$$

Then, by Lemma 3.3 we have

$$
\lim _{t \rightarrow \infty} X(t)<\infty \quad \text { a.s. }
$$

By the stochastic comparison theorem we get

$$
\int_{0}^{t}\left(\frac{\mu}{\mu+\phi}-S(s)\right)^{2} d s<\infty \quad \text { a.s. }
$$

Next, we prove that $S(t)$ is uniformly continuous. Denote $x=(S, I, V, R)$ in (23). Noting that $(S(t), I(t), V(t), R(t)) \in \Gamma$, we can easily check that the coefficients of (1) satisfy the local Lipschitz and linear growth conditions. In addition,

$$
\sup _{t \geq 0}\left\{E{\sqrt{S^{2}(t)+I^{2}(t)+V^{2}(t)+R^{2}(t)}}^{p}\right\} \leq 1 .
$$

Then, by Lemma 3.4, $\int_{0}^{t} g(s, x(s)) d \tilde{B}(s)$ is almost uniformly continuous along every sample path. At the same time, $\int_{0}^{t} f(s, x(s)) d s$ is almost uniformly continuous along every sample path due to the boundedness of $x$. So $x(t)$ is uniformly continuous, and hence $S(t)$ is uniformly continuous. Then by applying Lemma 3.5 to (24) we get

$$
\lim _{t \rightarrow \infty} S(t)=\frac{\mu}{\mu+\phi} \quad \text { a.s. }
$$

By the L'Hospital rule,

$$
\lim _{t \rightarrow \infty} R(t)=\lim _{t \rightarrow \infty} R(0) e^{-\mu t}+\lim _{t \rightarrow \infty} \gamma \int_{0}^{t} I(s) e^{-\mu(t-s)} d s=\frac{\gamma}{\mu} \lim _{t \rightarrow \infty} I(t)=0 \quad \text { a.s. }
$$

Then

$$
\lim _{t \rightarrow \infty} V(t)=\frac{\phi}{\mu+\phi} \quad \text { a.s. }
$$

To this end, we show that the disease-free equilibrium point $\left(S_{0}, I_{0}, V_{0}, R_{0}\right)=\left(\frac{\mu}{\mu+\phi}, 0\right.$, $\left.\frac{\phi}{\mu+\phi}, 0\right)$ is almost surely stable.

Remark 3 Theorem 3.1 implies that the disease will go extinct with probability one. More specifically,

- (19) and (20) show us that large noise will exponentially suppress the epidemic from prevailing.

- By (21), when the noise is small, $R_{0}^{S}<1$ is sufficient for ensuring the infectious class to go extinct. This, together with Theorem 2.1, leads to the conclusion that $R_{0}^{S}$ can be considered as the threshold whose value above one or below one completely determines the persistence and extinction of the disease in case that the noise is small. 
Remark 4 Note that $\frac{\beta}{\mu+\gamma}+\frac{(1+\eta) \sigma^{2}}{2} \leq \frac{1}{1+\eta}$ implies $\frac{\beta}{\mu+\gamma}<\frac{1}{1+\eta} \leq 1$. Then, it follows that

$$
\begin{aligned}
& \beta \frac{\mu+\eta \phi}{\mu+\phi}-(\mu+\gamma)-\frac{\mu^{2} \sigma^{2}}{2(\mu+\phi+\beta)^{2}} \\
& \quad=(\mu+\gamma)\left[\frac{\beta}{\mu+\gamma} \frac{\mu+\eta \phi}{\mu+\phi}-1-\frac{\mu^{2} \sigma^{2}}{2(\mu+\gamma)(\mu+\phi+\beta)^{2}}\right] \\
& \quad \leq(\mu+\gamma)\left[\frac{\beta}{\mu+\gamma}-1\right]<0 .
\end{aligned}
$$

Therefore, Theorem 3.1 gives the weakened conditions for extinction of the infective class than those in $[10,11]$.

\section{Extended results: threshold of the stochastic SIVS epidemic model}

To model the disease dynamic, when we suppose that the vaccination loses its effect at a proportional rate $\theta$, and a fraction $\lambda$ of infective goes back in the susceptible class, then model (1) will be rewritten as the model considered by Tornatore et al. [12]. This model has the form

$$
\left\{\begin{array}{l}
d S=[\mu-(\mu+\phi) S-\beta S I+\lambda I+\theta V] d t-\sigma S I d B(t), \\
d I=[\beta(S+\eta V) I-(\mu+\lambda) I] d t+\sigma(S+\eta V) I d B(t), \\
d V=(\phi S-\eta \beta V I-(\mu+\theta) V) d t-\sigma \eta V I d B(t) .
\end{array}\right.
$$

The existence of the solution and stability of disease-free equilibrium have been studied by Tornatore et al. [12]. However, the questions $A$ and $B$ are still left to be studied for this model. In the following, we will mainly establish the threshold result for model (25). Without loss of generality, we study the model on the positive invariant set

$$
\widetilde{\Gamma}=\{(S, I, V): S>0, I>0, V>0, S+I+V=1 \text { a.s. }\} .
$$

From (25) we compute that

$$
\begin{aligned}
S(t)+ & \eta V(t) \\
= & \frac{\mu+\theta+\eta \phi}{\mu+\phi+\theta}-\eta I(t)+(1-\eta)\left[S(0)-\frac{\mu+\theta}{\mu+\phi+\theta}\right] e^{-(\mu+\phi+\theta) t} \\
& -\beta(1-\eta) \int_{0}^{t} e^{-(\mu+\phi+\theta)(t-s)} S(s) I(s) d s+(\lambda-\theta)(1-\eta) \int_{0}^{t} e^{-(\mu+\phi+\theta)(t-s)} I(s) d s \\
& -\sigma(1-\eta) \int_{0}^{t} e^{-(\mu+\phi+\theta)(t-s)} S(s) I(s) d B(s) .
\end{aligned}
$$

By defining

$$
\tilde{R}=\frac{1}{\mu+\lambda}\left[\beta \frac{\mu+\theta+\eta \phi}{\mu+\phi+\theta}-\frac{\sigma^{2}}{2}\left(\frac{\mu+\theta+\eta \phi}{\mu+\phi+\theta}\right)^{2}\right]
$$

we get the following theorem.

Theorem 4.1 Let $(S(t), I(t), V(t))$ be a solution of system (25) with initial value $(S(0), I(0)$, $V(0)) \in \widetilde{\Gamma}$. 

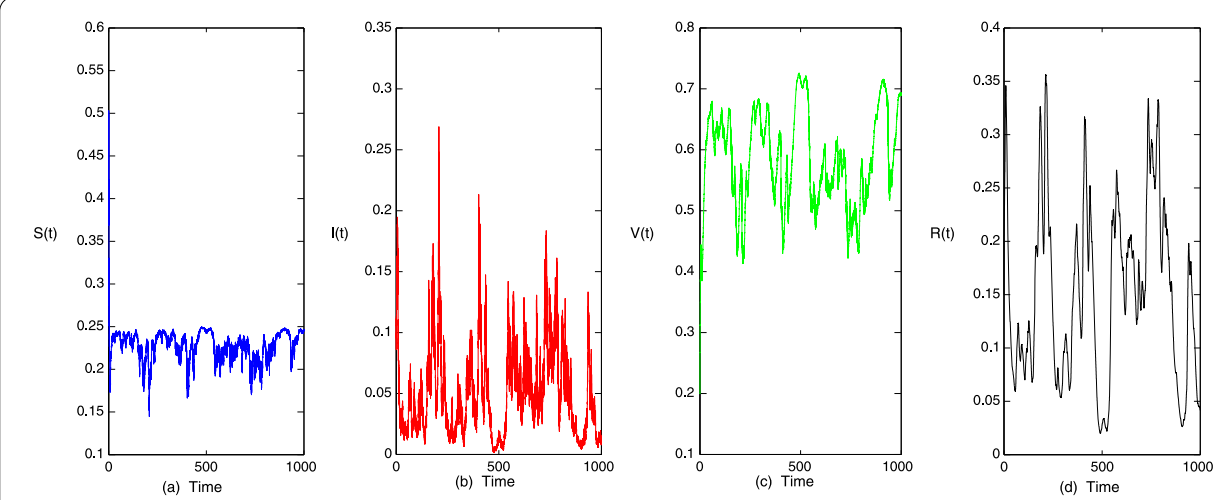

Figure 1 Simulations for the SDE model (1) with the parameters in Example 1.
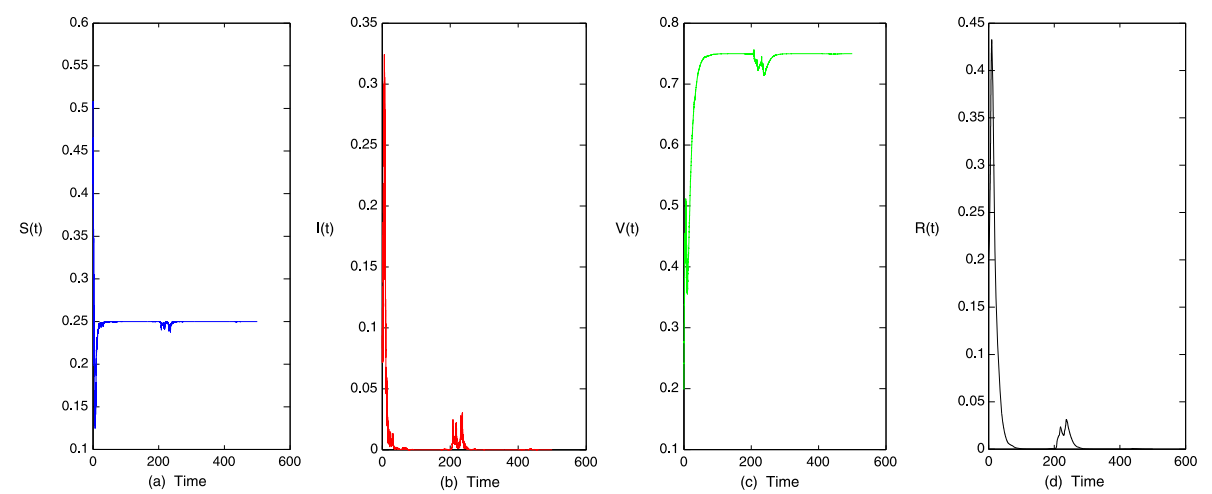

Figure 2 Simulations for the SDE model (1) with the parameters in Example 2.

(I) If $\tilde{R}>1$, then the disease will be persistent in mean.

(II) If $\sigma^{2} \frac{\mu+\theta+\eta \phi}{\mu+\phi+\theta} \leq \mu+\lambda$ and $\tilde{R}<1$, then the disease almost surely exponentially dies out.

The proof of Theorem 4.1 is omitted for similarity. Clearly, under the mild extra condition $\sigma^{2} \frac{\mu+\theta+\eta \phi}{\mu+\phi+\theta} \leq \mu+\lambda$, the disease will die out exponentially if $\tilde{R}<1$, and the disease will be persistent in mean if $\tilde{R}>1$. So we consider $\tilde{R}$ as a threshold of model (25).

\section{Computer simulations}

In this section, we will deal with two examples with simulation by the method proposed in [17] to show the results obtained in this paper.

Example 1 In model (1), we set $\beta=0.8, \mu=0.1, \gamma=0.3, \eta=0.5, \phi=0.3$, and $\sigma=0.4$. The initial value is $(S(0), I(0), V(0), R(0))=(0.5,0.1,0.2,0.15)$.

We compute that

$$
R_{0}^{S}=\frac{1}{\mu+\gamma}\left[\beta \frac{\mu+\eta \phi}{\mu+\phi}-\frac{\sigma^{2}}{2}\left(\frac{\mu+\eta \phi}{\mu+\phi}\right)^{2}\right]=1.1718>1 .
$$

Then Theorem 2.1 implies that the system will persist in time average, as shown by the following four pictures in Figure 1. 
Example 2 Let the noise intensity $\sigma=0.8$, and all the other parameters be the same as in Example 1.

We can compute that $\frac{1}{\mu+\gamma}\left[\beta \frac{\mu+\eta \phi}{\mu+\phi}\right]=1.25>1$. By the results given in $[10,11]$, we cannot be sure that the disease will go extinct or not. However, note that $R_{0}^{S}=0.9375<1$, Theorem 3.1 shows that the disease will almost surely go extinct, which means that large noise may greatly change the properties of the epidemic models. Furthermore, by Theorem 3.2 the disease-free equilibrium $(0.25,0,0.75,0)$ is stable with probability one. The computer simulations of Figure 2 support this.

\section{Competing interests}

The authors declare that they have no competing interests.

\section{Authors' contributions}

DZ conceived and proved the main results. SY performed the simulations and gave valuable discussions. Both authors read and approved the manuscript.

\section{Acknowledgements}

This work was supported by NSFC (No. 11271260, 11501148, 11171216), The Hujiang Foundation of China (B14005), and Shanghai Leading Academic Discipline Project (No. XTKX2012).

Received: 22 January 2016 Accepted: 21 October 2016 Published online: 28 October 2016

\section{References}

1. Xiao, D, Ruan, S: Global analysis of an epidemic model with nonmonotone incidence rate. Math. Biosci. 208, 419-429 (2007)

2. Gray, A, Greenhalgh, D, Hu, L, Mao, X, Pan, J: A stochastic differential equation SIS epidemic model. SIAM J. Appl. Math. 71, 876-902 (2011)

3. Lahrouz, A, Omari, L: Extinction and stationary distribution of a stochastic SIRS epidemic model with non-linear incidence. Stat. Probab. Lett. 83, 960-968 (2013)

4. Witbooi, PJ: Stability of an SEIR epidemic model with independent stochastic perturbations. Physica A 392, 4928-4936 (2013)

5. Lin, Y, Jiang, D, Wang, S: Stationary distribution of a stochastic SIS epidemic model with vaccination. Physica A 394 , 187-197 (2014)

6. Ji, C, Jiang, D: Threshold behaviour of a stochastic SIR model. Appl. Math. Model. 38, 5067-5079 (2014)

7. Zhao, Y, Jiang, D: The threshold of a stochastic SIRS epidemic model with saturated incidence. Appl. Math. Lett. 34, 90-93 (2014)

8. Chichigina, O, Valenti, D, Spagnolo, B: A simple noise model with memory for biological system. Fluct. Noise Lett. 52, 243-250 (2005)

9. Spagnolo, B, Valenti, D, Fiasconaro, A: Noise in ecosystem: a short review. Math. Biosci. Eng. 1, 185-211 (2004)

10. Tornatore, E, Buccellato, SM, Vetro, P: SIVR epidemic model with stochastic perturbation. Neural Comput. Appl. 24, 309-315 (2014)

11. Witbooi, PJ, Muller, GE, Van Schalkwyk, GJ: Vaccination control in a stochastic SVIR epidemic model. Comput. Math. Methods Med. 2015, Article ID 271654 (2015). doi:10.1155/2015/271654

12. Tornatore, E, Buccellato, SM, Vetro, P: On a stochastic disease model with vaccination. Rend. Circ. Mat. Palermo 55 , 223-240 (2006)

13. Mao, X: Almost sure asymptotic bounds for a class of stochastic differential equations. Stoch. Stoch. Rep. 41, 57-69 (1992)

14. Mao, X: Stochastic Differential Equations and Applications. Horwood, Chichester (1997)

15. Mao, X: Stochastic versions of the LaSalle theorem. J. Differ. Equ. 153, 175-195 (1999)

16. Jiang, D, Shi, N, Zhao, Y: Existence, uniqueness, and global stability of positive solutions to the food-limited population model with random perturbation. Math. Comput. Model. 42, 651-658 (2005)

17. Higham, DJ: An algorithmic introduction to numerical simulation of stochastic differential equations. SIAM Rev. 43, 525-546 (2001) 